\title{
A Survey of Revision Approaches in Description Logics
}

\author{
Guilin Qi ${ }^{1}$ and Fangkai Yang ${ }^{2}$ \\ 1 Institute AIFB \\ Universität Karlsruhe (TH), Germany \\ gqi@aifb.uni-karlsruhe.de \\ 2 Fangkai Yang \\ Multi-Agent System Lab \\ University of Science and Technology of China, Hefei, China \\ fkyang@mail.ustc.edu.cn
}

\begin{abstract}
Revision of a Description Logic-based ontology to incorporate newly received information consistently is an important problem for the lifecycle of ontologies. Many approaches in the theory of belief revision have been applied to deal with this problem and most of them focus on the postulate or logical properties of a revision operator in Description Logics (DLs). However, there is no coherent view on how to characterize a revision operator in DLs. In this paper, we lay bare the assumptions underlying different approaches for revision in DLs and propose some criteria to compare them. Based on the analysis, we give our definition of a revision operator in DLs and point out some open problems.
\end{abstract}

Key words: Description Logic, Ontology Evolution, Belief Revision

\section{Introduction}

Ontologies play a crucial role for the success of the Semantic Web [3]. One of the challenging problems for the development of ontology is ontology evolution, which is defined as the timely adaptation of an ontology to the arisen changes and the consistent management of these changes [9,24]. One of the center problem during ontology evolution is inconsistency handling. There are various forms of inconsistencies, such as structural inconsistency, logical inconsistency and userdefined inconsistency. Among them, logical inconsistency has received lots of attention, where ontologies are represented by logical theories, such as description logics (DLs) $[6,10,9]$.

Theory of belief change in propositional logic or first-order logic deals with logical inconsistency resulting from revising a knowledge base by newly received information. There are three types of belief change, i.e. expansion, contraction and revision. Expansion is simply to add a formula to a knowledge base; contraction requires to consistently remove a formula from a knowledge base and revision is the problem of accommodating a new formula to a knowledge base consistently. Alchourrón, Gardenfors and Markinson propose a set of postulates 
to characterize each belief change operator (see [8] for their work). The application of AGM's theory to description logics is not trivial because it is based on the assumptions that generally fail for DLs [5]. In [5,6], the basic AGM postulates for contraction are generalized to DLs and the feasibility of applying the generalized AGM theory of contraction to DLs and OWL is studied. However, they do not consider the application of AGM postulates for revision in DLs. It is argued in [21] that the recovery postulate for contraction is not intuitive and another postulate, called relevance, is introduced to replace it. According to [6],

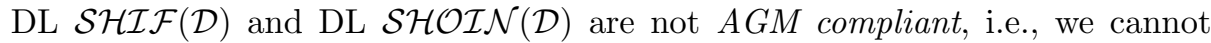
define a contraction operator that satisfies the generalized AGM postulates in these DLs.

In AGM's theory, epistemic states are represented by belief sets, i.e., a set of formulas that are closed under logical consequence. However, in a belief set, there is no difference between the explicitly represented knowledge (i.e., those formulas that are stored in the databases) and the implicitly represented one (i.e., those formulas that are implied by the explicitly represented one). Therefore, many researchers turn to use belief bases, i.e., a finite set of formulas that may not be closed under logical consequence, to represent epistemic sates of an agent (see [7, $16,13,14])$. In [4], the authors argue that in the context of ontology evolution, it is more natural to differentiate the explicit knowledge and implied knowledge in an ontology. They then propose a set of postulates for contraction operators by dropping the assumption that the result of contraction is an ontology which is closed under logical consequence. They also define a set of postulates for revision by introducing negation of axioms. In [11], Hansson's semi-revision operator is applied to deal with the revision problem in DLs and an algorithm is given to implement the revision operator. However, semi-revision operator does not guarantee the success postulate, which says that the newly received information will be kept after revision. Therefore, in [22], two revised semi-revision operators are given to guarantee the property of (weak) success. The disadvantage of their approaches is that they are dependent on the syntactical forms of axioms and so are not fine-grained.

Most of important work on revision in DLs is based on AGM's theory. However, compared with mature research in the area of belief revision, e.g., AGM's theory, some of them are not well justified and also deviate from AGM paradigms, leading to a misconception that AGM theory is unsuitable for DLs. Therefore, it is important to analyze these approaches to lay bare the assumptions underlying them. The forthcoming Semantic Web constitutes an ideal application scenario for formal logic and traditional commonsense reasoning approaches. Therefore, many people from traditional knowledge representation and reasoning community are interested in apply the work on belief revision to DLs. But they may not be familiar with the literature and find it hard to know where to start. So, another motivation of this work is to report the latest progress of the work on revision in DLs and point out some future directions to facilitate their study.

In this paper, we investigate the problem of revision in description logics. We mainly consider the following questions: 
- What is a revision operator in description logics? This is a fundamental question to be answered.

- What postulates should a revision operator satisfy? This is the main question that will be discussed in this paper. This question is closely related to the first question.

\section{Description logics}

In this section, we briefly introduce Description Logics (DLs). For more details, we refer the reader to [2]. A DL-based knowledge base (or ontology) is a pair $K=(\mathcal{T}, \mathcal{A})$, where $\mathcal{T}$, called the TBox, is a set of concept axioms and role axioms, and $\mathcal{A}$, called the $\mathrm{ABox}$, is a set of assertional axioms. Concept axioms have the form $C \sqsubseteq D$ where $C$ and $D$ are concept expressions, and role axioms have the form $R \sqsubseteq S$, where $R$ and $S$ are role expressions. Assertional axioms have the form $C(a)$, where $C$ is a concept and $a$ is an individual name, or have the form $R(a, b)$, where $R$ is a role and $a$ and $b$ are individual names.

In DLs, an interpretation $\mathcal{I}=\left(\triangle^{\mathcal{I}}, \cdot^{\mathcal{I}}\right)$ consists of a non-empty domain set $\triangle^{\mathcal{I}}$ and an interpretation function ${ }^{\mathcal{I}}$, which maps from individuals, concepts and roles to elements of the domain, subsets of the domain and binary relations on the domain, respectively. Given an interpretation $\mathcal{I}$, we say that $\mathcal{I}$ satisfies a concept

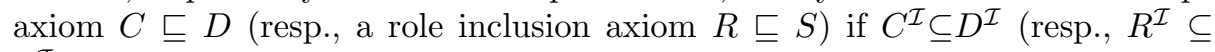
$\left.S^{\mathcal{I}}\right)$. Furthermore, $\mathcal{I}$ satisfies a concept assertion $C(a)$ (resp., a role assertion $R(a, b))$ if $a^{\mathcal{I}} \in C^{\mathcal{I}}$ (resp., $\left.\left(a^{\mathcal{I}}, b^{\mathcal{I}}\right) \in R^{\mathcal{I}}\right)$. An interpretation $\mathcal{I}$ is called a model of an ontology $K$, iff it satisfies each axiom in $K$. A concept name $C$ in an ontology $K$, is unsatisfiable if for each model $\mathcal{I}$ of $K, C^{\mathcal{I}}=\emptyset$. An ontology $K$ is incoherent if there exists an unsatisfiable concept name in $K$. An ontology $K$ is inconsistent iff it has no model.

\section{A Survey of Revision Approaches in DLs}

In this section, we give a survey of existing approaches for revision in DLs. We can roughly classify these approaches into two families: AGM-based approaches and non-AGM-based approaches. In the following, we introduce them in turn.

\subsection{AGM-based approaches}

The importance of applying AGM theory on belief change to terminological systems has not been fully recognized until recent years. The first work that tries to apply AGM theory to description logics may be traced back to Nebel's thesis [18] published in 1990. He first gives five general principles for knowledge base revision in a logical language. He then argues that revision of a TBox should only be applied to definition of an atomic concept. Finally, a revision operator is defined to revise concept definitions that satisfies all the principles. However, there is no further work following his idea. 
Recently, there is an increasing interest in applying belief revision theory to handle inconsistency during ontology evolution. In $[5,6]$, some work has been done to analyze the feasibility of applying AGM postulates for contraction to DLs (the underlying logic is more general than DLs in their work). Their work is based on the coherence model, i.e. the revised knowledge base should be knowledge set which is knowledge base closed under logical consequence (see K-1 below). They reformulate AGM postulates to a general logic. Here we restrict the logic to a DL. Suppose ' - ' is the operation of contraction which refers to the consistent removal of a piece of information from a knowledge base. Let $\mathrm{Cn}$ be consequence relation in a DL language, their postulates are given as follows:

(K-1) Closure: $\operatorname{Cn}(K-X)=K-X$

(K-2) Inclusion: $K-X \subseteq \mathrm{Cn}(K)$

(K-3) Vacuity: If $X \nsubseteq \mathrm{Cn}(K)$, then $K-X=\mathrm{Cn}(K)$

(K-4) Success: If $X \nsubseteq \mathrm{Cn}(\emptyset)$, then $X \nsubseteq \mathrm{Cn}(K-X)$

(K-5) Preservation: If $\operatorname{Cn}(X)=\operatorname{Cn}(Y)$, then $K-X=K-Y$

(K-6) Recovery: $K \subseteq \operatorname{Cn}((K-X) \cup X)$

They then define a logic to be AGM-compliant iff a contraction operator that satisfies the generalized AGM postulates given above can be defined in the given logic. It has been shown that some important DLs, such as $\mathcal{S H \mathcal { I }}$ and $\mathcal{S H O I N}$, are not AGM compliant. They also show that by adding role operators to the $\mathcal{A L}$ family we can obtain some AGM-compliant DLs, such as $\mathcal{A L C O}{ }^{\neg, \sqcap, \sqcup, ~}$ and $\mathcal{A} \mathcal{L C}\urcorner, \sqcap, \sqcup$ with empty ABox.

It is argued in [21] that the recovery postulate for contraction is not intuitive according to the discussion in the literature and the authors introduce another postulate, called relevance, which is originally defined in [12]:

Relevance: If $\psi \in K$ and $\psi \notin K-\phi$, then there is a set $K^{\prime}$ such that $K-\phi \subseteq K^{\prime} \subseteq K$ and that $\phi \notin \operatorname{Cn}\left(K^{\prime}\right)$, but $\phi \in \operatorname{Cn}\left(K^{\prime} \cup\{\psi\}\right)$.

They have shown that there exists a contraction operator for DL $\mathcal{S H} \mathcal{H} \mathcal{F}(\mathcal{D})$ and DL $\mathcal{S H O I N}(\mathcal{D})$ that satisfies generalized AGM postulates with Relevance instead of Recovery.

None of the above work considers the explicit construction of a contraction operator that satisfies their postulates, which makes the postulates not fully justified. In contrast, in AGM's work, their postulates are well justified by some concrete contraction operators. Furthermore, they did not consider the application of AGM postulates for revision in DLs. One may wonder if we can define a revision operator by a contraction operator via the Levi identity, i.e., $K \circ X=C n((K-\neg X) \cup X)$. The problem is that Levi identity is not applicable for most DLs [6] because negation of a DL axiom is not well-defined.

In [4], the authors advocate that in the context of ontology evolution, it is more natural to differentiate the explicit knowledge and implied knowledge in an ontology. They then propose a set of postulates for contraction by dropping the assumption that a knowledge base or an ontology is closed under logical consequence. They also define a set of postulates for revision by introducing axiom negation. Let $K$ and $X$ be two DL knowledge bases, and $\circ$ be a revision operator, then we have the following postulates: 
$(\mathrm{O}+1) X \subseteq K \circ X$.

$(\mathrm{O}+2)$ If $K \cup X$ is consistent, then $K \circ X=K \cup X$.

$(\mathrm{O}+3)$ If $X$ is consistent, then $K \circ X$ is also consistent.

$(\mathrm{O}+4)$ If $X \equiv Y$, then $K \circ X \equiv K \circ Y$.

Plus the following postulate which is defined by a contraction operator:

$(\mathrm{O}+5)(K \circ X) \cap K \equiv K-\neg X$, where the negation of an axiom has two different definitions (consistency-negation and coherence-negation) given in [4].

$(\mathrm{O}+1)$ says that newly received information must appear in the revised ontology. $(\mathrm{O}+2)$ is one of the postulates for minimal change. $(\mathrm{O}+3)$ states that the revised ontology should be consistent if the newly received one is. $(\mathrm{O}+4)$ is the postulate for syntax-irrelevance.

The postulates $(\mathrm{O}+1)-(\mathrm{O}+5)$ give some good insights on how a rational revision operator should behave. For example, they introduce negation of an axiom to define a revision operator by a contraction operator and they also point out that there are two kinds of logical contradictions that a revision operator need to deal with. The authors claim that their postulates correspond to postulates for revision given in [1]. Unfortunately, their reformulation of AGM postulates deviate the original idea of AGM theory. They ignore one important assumption underlying AGM postulates for revision given in [1]: the result of revision should be a knowledge set (a set of formulas which are closed under logical consequence), instead of a knowledge base. Indeed, according to $(\mathrm{O}+2)$, the result of revision should be a DL knowledge base which may not be a knowledge set. Therefore, their postulates cannot really capture the original meaning of AGM postulates for revision. Furthermore, in AGM theory, most of revision operators satisfying AGM postulates, such as the partial meet revision operator, are defined by disjunction of some knowledge bases containing the newly received formula. The reason for using disjunction is that when revising a knowledge base, there are usually several alternative ways to resolve an inconsistency, and one does not know which solution is the best one. According to $(\mathrm{O}+1)$ and $(\mathrm{O}+2)$, the result of revision must be a single ontology which contains the new ontology $X$. Since disjunction of ontologies is not allowed in DLs, it is impossible for them to keep all or some of the possible ontologies which are subsets of the original ontology and are consistent with the new ontology, so their revision operator will choose an arbitrary one if there is no extra information to guide which one should be chosen.

Another serious problem of their work is that it is not clear how their postulates ensure the principle of minimal change. In AGM theory, to demonstrate that their postulates ensure the principle of minimal change, they give a representation theorem showing that a revision operator satisfies their postulates iff it can be defined by an epistemic entrenchment relation on formulas. There is no discussion on such correspondence in [4]. Furthermore, they do not give a revision operator that satisfies their postulates to validate their postulates.

In parallel to the work in [4], Qi et.al. in [20] propose a set of postulates for knowledge base revision in DLs based on reformulated AGM postulates given in [16]. They define a revision operator as a mapping from a pair of DL knowledge 
bases to a disjunctive $D L$ knowledge base. A disjunctive $D L$ knowledge base $\mathcal{K}$ (or DKB), defined in [17], is a set of DL knowledge bases. An interpretation is a model of a disjunctive DL knowledge base $\mathcal{K}$ iff it is a model of one of DL knowledge bases in $\mathcal{K}$. Given a knowledge base $K$ (resp. a disjunctive knowledge base $\mathcal{K}$ ), we use $M(K)$ (resp. $M(\mathcal{K}))$ to denote the set of all its models. Let $K$ and $K^{\prime}$ be DL knowledge bases. We introduce their postulates as follows:

(G1) $M\left(K \circ K^{\prime}\right) \subseteq M(\phi)$ for all $\phi \in K^{\prime}$

(G2) If $M(K) \cap M\left(K^{\prime}\right) \neq \emptyset$, then $M\left(K \circ K^{\prime}\right)=M(K) \cap M\left(K^{\prime}\right)$

(G3) If $K^{\prime}$ is consistent, then $M\left(K \circ K^{\prime}\right) \neq \emptyset$

(G4) If $M(K)=M\left(K_{1}\right)$ and $M\left(K^{\prime}\right)=M\left(K_{2}\right)$, then $M\left(K \circ K^{\prime}\right)=M\left(K_{1} \circ K_{2}\right)$.

(G5) $M\left(K \circ K^{\prime}\right) \cap M\left(K^{\prime \prime}\right) \subseteq M\left(K \circ\left(K^{\prime} \cup K^{\prime \prime}\right)\right)$

(G6) If $M\left(K \circ K^{\prime}\right) \cap M\left(K^{\prime \prime}\right)$ is not empty, then $M\left(K \circ\left(K^{\prime} \cup K^{\prime \prime}\right)\right) \subseteq M\left(K \circ K^{\prime}\right) \cap M\left(K^{\prime \prime}\right)$

(G1) guarantees that the new information is inferred from each revised knowledge base. (G2) requires that when there is no conflict between $K$ and $K^{\prime}$, we do not need to change the original knowledge base. (G3) is a condition preventing a revision from introducing unwarranted inconsistency. (G1)-(G3) corresponds to $(\mathrm{O}+1)-(\mathrm{O}+3)$. (G4) requires that the revision operator is independent of the syntactical forms of both the original DL knowledge base and the newly received one. It is stronger than $\mathrm{O}+4$. However, this postulate is not always advisable because we may want to keep the structure of DL axioms after revision. In [20], a weaker version of (G4) is proposed:

(G4) If $K_{1}$ and $K_{2}$ are element-equivalent and $M\left(K_{1}^{\prime}\right)=M\left(K_{2}^{\prime}\right)$, then $M\left(K_{1} \circ K_{1}^{\prime}\right)$ $=M\left(K_{2} \circ K_{2}^{\prime}\right)$, where $K_{1}$ and $K_{2}$ are said to be element-equivalent iff there is a bijectin $f$ from $K_{1}$ to $K_{2}$ such that for every $\phi$ in $K_{1}, M(f(\phi))=M(\phi)$.

Similar to the work in [16], we can show a representation theorem for the postulates (G1)-(G6) or (G1)-(G3), G(4)', (G5) and (G6). We first define a faithful assignment.

Definition 1. Let $K$ and $K^{\prime}$ be $D L$ knowledge bases and $\Omega$ be the set of all interpretations in the considered DL language, a total pre-order $\preceq_{K}$ on $\Omega$, associated with $K$, is said to be a faithful assignment if the following conditions hold:

1. if $\mathcal{I}, \mathcal{I}^{\prime}=K$, then $\mathcal{I} \prec_{K} \mathcal{I}^{\prime}$ does not hold.

2. if $\mathcal{I} \models K$ and $\mathcal{I}^{\prime} \not \models K$, then $\mathcal{I} \prec_{K} \mathcal{I}^{\prime}$ holds.

3. if $K \equiv K^{\prime}$, then $\preceq_{K}=\preceq_{K^{\prime}}$.

Furthermore, $\preceq_{K}$ is said to be a weak faithful assignment if it satisfies condition 1 and condition 2 above and the following condition: if $K$ and $K^{\prime}$ are element equivalent, then $\preceq_{K}=\preceq_{K^{\prime}}$.

The following theorem establishes the correspondence between the set of postulates and the (weak) faithful assignment.

Theorem 1. A revision operator o satisfies the postulates (G1)-(G6) (resp. (G1)-(G3), G(4)', (G5) and (G6)) iff for any DL knowledge base K, there exists a faithful assignment (resp. weak faithful assignment) $\preceq_{K}$ such that $\operatorname{Mod}(K \circ$ $\left.K^{\prime}\right)=\min \left(\operatorname{Mod}\left(K^{\prime}\right), \preceq_{K}\right)$, where $\operatorname{Mod}(K)$ is the set of all models of $K$. 
Two concrete revision operators were proposed in [20], both satisfying (G1)(G3), (G4)', (G5) and (G6). When revising an ontology by another ontology, they propose to circumscribe minimal number of individuals that are responsible for the conflict from axioms in the TBox and/or minimal number of axioms from the ABox in the original ontology to restore consistency. Therefore, their revision operators are more fine-grained than those resolve inconsistency by deleting the whole axioms. One weakness of their work is that they consider only inconsistency when ontology evolves and do not consider incoherence. Moreover, their revision operators deal with only inconsistencies arising due to objects being explicitly introduced in the ABox.

\subsection{Non-AGM-based approach}

In [11], Hansson's semi-revision operator is adapted to DLs and an algorithm is given to implement the revision operator. However, a semi-revision operator does not guarantee that the newly received information will be kept after revision (i.e., (R1) in [4] or (G1) in [20]). Therefore, in [22], two revised semi-revision operators are given to guarantee this property. Unlike the approach in [20], their approach deletes the whole axiom in ABox and TBox to restore consistency. One of their revision operator, called kernel revision without negation can be equivalently defined by some postulates. Let $K$ be an ontology, $\phi$ and $\psi$ be axioms. Then $\circ$ is a kernel revision operator without negation iff it satisfies the following postulates: [success] $\phi \in K \circ \phi$

[weak consistency] If $\phi$ is consistent, then $K \circ \phi$ is consistent

[inclusion] $K \circ \phi \subseteq K \cup\{\phi\}$

[core-retainment] If $\psi \in K$ and $\psi \notin K \circ \phi$ then there is at least a consistent subset $K^{\prime}$ of $K \cup\{\phi\}$ such that $K \cup\{\psi\}$ is inconsistent.

[pre-expansion] $(K \cup\{\phi\}) \circ \phi=K \circ \phi$

The result of the kernel revision operator is a single ontology according to postulate [success]. This postulate corresponds to postulate $(\mathrm{O}+1)$. Together with postulate [success], postulate [inclusion] says that the revision operator takes the subset of the original ontology and add the new axiom $\phi$ to it. Postulate [core-retainment] states that if an axiom is deleted after revision, then this axiom must belong to a sub-ontology that is in conflict with the new axiom. By [inclusion] and [core-retainment], we can infer that the kernel revision operator also satisfies the following postulate which is similar to [O+2]: If $K \cup\{\phi\}$ is consistent, then $K \circ \phi=K \cup\{\phi\}$.

The work in [9] describes a process to support the consistent evolution of OWL DL based ontologies, which ensures that the consistency of an ontology is preserved when changes are applied to the ontology. Two algorithms were given to find minimal inconsistent sub-ontologies and maximal consistent subontologies. There is no guarantee that their algorithms can find all the minimal inconsistent sub-ontologies or maximal consistent sub-ontologies, although they can efficiently find one minimal inconsistent sub-ontology and one maximal consistent sub-ontology. They consider only adding one axiom (instead of an ontology) to an ontology consistently. 


\subsection{Comparison}

There are many discussions on how to apply existing work on belief revision to description logics. In this section, we propose some criteria to give a comparison among them.

- Implementation (IM): Considering the vision of the Semantic Web, we should give more emphasis on implementation of a revision approach. We consider two issues: is there an algorithm for the operator and has the algorithm been implemented?

- Minimal Change (MC):When we revise a knowledge base, a natural requirement is that the operator should keep as much original information as possible.

- Preservation of Structure (PS): We say that a revision operator preserves the structure of an axiom in an ontology if we can only remove or change some (or all) of its concept(s) or instance(s).

- Language Dependence ( $L D)$ : Some revision operator can be applied to any DL, such as those defined in [22]. However, utilizing certain special features of DL may lead to fine-grained revision operators and can return desirable results efficiently for certain useful languages, although these operators are not general enough for all family of DLs,.

- Uniqueness of the Result (UR): Disjunction among axioms or ontologies is not allowed in DLs. Therefore, a revision operator either results in a single ontology or a set of ontologies. When an inconsistency or incoherence is encountered, there are usually several alternative ways to resolve it. To return a unique ontology, the revision operator will have to select an arbitrary solution from those possible ones. Alternative, the revision operator can result in a set of ontologies and leave decision of which one should be selected to the user.

- Inconsistency vs. Incoherence (II): According to the discussion in [4], incoherence is a potential cause of inconsistency but it does not provide the classical sense of the inconsistency. It is interesting to know if a revision operator can resolve incoherence or inconsistency (or both).

- Support for ABox,TBox (SU): In DLs, incoherence usually occurs in TBoxes, so a revising operator that deals with incoherence only need to support TBox. Although inconsistency can occurs in TBoxes for some DLs, it is caused by conflict between TBox and ABox in most cases. So a revision operator dealing with inconsistency usually supports both TBox and ABox. Since terminology axioms may make the revision very complex, we may restrict the input ontology to an ABox.

- Complexity (CO): Reasoning with expressive DLs is intractable for standard reasoning tasks. Often the approaches for dealing with inconsistencies introduce an additional level of complexity. In order to assure practicability, these complexity issues need to be taken into account.

- Interactivity, user involvement (IU): To revise an ontology w.r.t. another one, we usually have different alternative ways to resolve the conflict. As we 
have discussed before, it may be desirable to ask the user to decide which solution is the best one. Therefore, a semi-automated revision approach seems to be promising in practice.

\begin{tabular}{|l|r|r|r|r|r|}
\hline Criteria & Approach 1 & Approach 2 & Approach 3 & Approach 4 & Approach 5 \\
\hline IM & n.a. & n.a. & no & no & yes \\
MC & unknown & unknown & yes & yes & yes \\
PS & n.a. & n.a. & partially & yes & yes \\
LK & see [6] & all DLs & DLs $(\mathcal{O})$ & all DLs & all DLs \\
UR & unknown & yes & no & yes & yes \\
II & IS & both & IS & IS & IS \\
SU & both & both & both & both & both \\
IU & no & no & no & no & no \\
\hline
\end{tabular}

Table 1. Approach 1:approach in [6], Approach 2:approach in [4], Approach 3: approach in [20], Approach 4: approach in [22], Approach 5: approach in [9], n.a. means "not applicable". DLs $(\mathcal{O})$ in low 5 means DLs with nominals, and IS in row 7 means "inconsistency".

The results of comparison are compactly summarized in Table 1 . We do not list the approaches in [18], in [21], and in [11]. The approach in [18] was not followed afterwards, so we do not consider it. The approach in [21] is similar to the approach 1 in [6], so the result in Table 1 can be applied to it as well. The approach in [11] is not a revision operator in a strict sense because it does not satisfy the success postulate, which is a mandatory postulate for a revision operator. Furthermore, this approach is similar to approach 4 in [22]. We also do not discuss complexity of different approaches because most of them do not have an algorithm to implement them.

According to Table 1, the syntax-based revision approaches (i.e. approach 4 and approach 5) seem to be more promising than other approaches. These approaches can be very easily generalized to deal with incoherence as well. For example, it is possible to apply the approaches for repairing terminologies in [19, $23]$ to revise terminologies. Approach 1 and approach 2 apply AGM postulates to DLs, but their approaches suffer from two problems. First, it is not clear how their postulates can ensure minimal change. Second, there is no concrete revision operator given to validate their postulates. Approach 3 apply reformulated AGM postulates to DLs and also give two concrete revision operators which are applicable to DLs with nominals. One of their operator, called refined-weakening based revision operator, requires to split axioms, so it does not preserve the structure of the axioms. The other revision operator, called weakening-based revision operator in [20], does not change the structure of the axioms. Both revision operators have not been implemented.

\section{Conclusion and Future Work}

In this paper, we gave a brief review of existing approaches for revision in DLbased ontologies. We also proposed a set of criteria to compare these approaches. 
Based on our analysis, A revision operator in description logics is an operation that maps an ordered pair of DL ontologies (or knowledge bases) to a set of ontologies such that each of the revised ontology should be consistent and can infer every axiom in the second ontology, and that can be constrained by a set of postulates. This definition of revision operator also partially answers the second question given in Section 1. That is, any ontology obtained by revision should be consistent and should infer every axiom in the new ontology. In our definition, coherence is not a mandatory requirement for a revision operator because even if an ontology is incoherent, it can be consistent and we can apply any DL reasoner to infer non-trivial conclusions from it, although some of the conclusions may not make sense. For the second question, there is no unique answer for it because there are different sets of postulates for a revision operator in DLs for different ways to resolve inconsistency or incoherence and different ways to ensure minimal change. The sets of postulates given in [20] and [22] capture the principle of minimal change. Therefore, they can be used to define a rational revision operator. The difference between the postulates in [20] and those in [22] is that the former characterizes those revision operators defined by a total pre-order whilst the latter characterizes those revision operators defined by an incision function which selects axioms to be deleted. Therefore, the former allows more fine-grained approaches for resolving inconsistency. In contrast, the sets of postulates given in [6] and [4] lack of justifications for minimal change and are hard to be used.

Some research directions can be drawn according to Table 1. For example, it would be interesting to know how postulates given in [6] and [4] ensure minimal change. It is also interesting to implement a revision tool which allows the user to make some decision.

\section{Acknowledgments}

The first author is partially supported by the EU in the IST project $\mathrm{NeOn}$ (EU IST-2006-027595, http://www.neon-project.org/).

\section{References}

1. Carlos E. Alchourrón, Peter Gärdenfors, and David Makinson. On the logic of theory change: Partial meet contraction and revision functions. J. Symb. Log., 50(2):510-530, 1985.

2. Franz Baader, Diego Calvanese, Deborah McGuinness, Daniele Nardi, and Peter Patel-Schneider. The Description Logic Handbook: Theory, implementation and application. Cambridge University Press, 2003.

3. Tim Berners-Lee, James Hendler, and Ora Lassila. The semantic web. Scientific American, 284(5):3443, 2001.

4. Giorgos Flouris, Zhisheng Huang, Jeff Z. Pan, Dimitris Plexousakis, and Holger Wache. Inconsistencies, negations and changes in ontologies. In Proc. of AAAI'06, pages 1295-1300, 2006. 
5. Giorgos Flouris, Dimitris Plexousakis, and Grigoris Antoniou. Generalizing the agm postulates: preliminary results and applications. In Proc. of $N M R$ '04, pages 171-179, 2004.

6. Giorgos Flouris, Dimitris Plexousakis, and Grigoris Antoniou. On applying the AGM theory to DLs and OWL. In Proc. of 4 th International Conference on Semantic Web, pages 216-231. 2005.

7. Andre Fuhrmann. Theory contraction through base contraction. Journal of Philosophical Logic, 20(2):175-203, 1991.

8. Peter Gardenfors. Knowledge in Flux-Modeling the Dynamic of Epistemic States. The MIT Press, Cambridge, Mass, 1988.

9. Peter Haase and Ljiljana Stojanovic. Consistent evolution of owl ontologies. In Proc. of ESWC'05, pages 182-197, 2005.

10. Peter Haase, Frank van Harmelen, Zhisheng Huang, Heiner Stuckenschmidt, and York Sure. A framework for handling inconsistency in changing ontologies. In Proc. of 4th International Semantic Web Conference (ISWC'05), pages 353-367. Springer, 2005.

11. Christian Halaschek-Wiener, Yarden Katz, and Bijan Parsia. Belief base revision for expressive description logics. In Proc. of OWL-ED'06. 2006.

12. Sven Ove Hansson. New operators for theory change. Theoria, 55:114-32, 1989.

13. Sven Ove Hansson. Reversing the levi identity. Journal of Philosophical Logic, 22(6):637-669, 1993.

14. Sven Ove Hansson. Semi-revision (invited paper). Journal of Applied Non-Classical Logics, 7(2):151-175, 1997.

15. Sven Ove Hansson. A Textbook of Belief Dynamics: Theory Change and Database Updating. Kluwer Academic Publishers, 1999.

16. Hirofumi Katsuno and Alberto O. Mendelzon. Propositional knowledge base revision and minimal change. Artif. Intell., 52(3):263-294, 1992.

17. Thomas Meyer, Kevin Lee, and Richard Booth. Knowledge integration for description logics. In Proc. of 20th National Conference on Artificial Intelligence (AAAI'05), pages 645-650. AAAI Press, 2005.

18. Bernhard Nebel. Reasoning and Revision in Hybrid Representation Systems, volume 422 of Lecture Notes in Computer Science. Springer, 1990.

19. Peter Plessers and Olga De Troyer. Resolving inconsistencies in evolving ontologies. In Proc. of ESWC'06, pages 200-214, 2006.

20. Guilin Qi, Weiru Liu, and David A. Bell. Knowledge base revision in description logics. In Proc. of JELIA'06, pages 386-398. Springer Verlag, 2006.

21. Márcio Moretto Ribeiro and Renata Wassermann. First steps towards revising ontologies. In Proceedings of the Second Workshop on Ontologies and their Applications (WONTO 2006), 2006.

22. Márcio Moretto Ribeiro and Renata Wassermann. Base revision in description logics - preliminary results. In Proceedings of the International Workshop on Ontology Dynamics (IWOD 2007), 2007.

23. Stefan Schlobach, Zhisheng Huang, Ronald Cornet, and Frank van Harmelen. Debugging incoherent terminologies. J. Autom. Reasoning, 39(3):317-349, 2007.

24. Ljiljana Stojanovic. Methods and Tools for Ontology Evolution. PhD thesis, University of Karlsruhe, 2004. 